\title{
CIRCUMSTELLAR MOLECULAR SPECTRA TOWARDS EVOLVED STARS
}

\author{
ERIC J. BAKKER \\ Department of Astronomy and W.J. McDonald Observatory, \\ University of Texas, Austin, TX 78712, USA
}

\begin{abstract}
In this paper we discuss the relevance of, and possible scientific gains which can be acquired from studying circumstellar molecular spectra toward evolved stars. Where can we expect circumstellar molecular spectra, why would we want to study these spectra, which molecules might be present, and what can we learn from these studies? We present an overview of reported detections, and discuss some of the results.
\end{abstract}

\section{Introduction}

Observational studies of the circumstellar environment (CSE) of evolved stars mainly concentrate on infrared emission from dust (peaking around $60 \mu \mathrm{m}$ ) and radio line emission of various molecular species (e.g. CO, HCN, $\mathrm{CN})$. Both tracers are very powerful in studying a large sample of stars, and the overall characteristics of the CSE, but, since they are in emission they lack positional information about the line forming region.

Other tracers of the CSE are infrared emission features (Polycyclic Aromatic Hydrocarbons, PAH; Unidentified Infrared Bands, UIR; circumstellar reddening). The physics of these latter tracers are not well understood and with our current knowledge it is not possible to extract detailed physical information from these tracers.

A different category of tracer is molecular spectra. Absorption spectra only probe a well defined pencil beam toward the continuum source, and are very well suited for detailed studies of the CSE. Emission spectra are rare and their occurrence is almost always connected with some kind of violent mechanism (e.g. shocks). Fortunately, the molecules for which circumstellar spectra have been detected are rather simple with at most three atoms (Table 1). The physics of these molecules is sufficiently well understood 
that they can be used to extract physical information about the line forming region (Sect. 1.4). An additional advantage of these molecules is that their electronic spectra fall in the ultraviolet and optical part of the spectrum and acquiring these spectra is relatively easy.

In this paper we discuss circumstellar molecular spectra with a strong emphasis on absorption line studies. We will ask ourself four question: where are they, why bother, which species, and what can we learn?

\subsection{WHERE CAN WE EXPECT CIRCUMSTELLAR SPECTRA?}

In the broadest sense we could argue that circumstellar absorption spectra are expected to be present in all cases where there is circumstellar matter between the observer and the continuum source. The continuum source is mostly the star. We therefore can expect molecular spectra towards RGB, AGB, post-AGB stars (Bakker et al. 1996/1997, Bakker \& Lambert, from now on Paper I, II, III), supernovae (Dalgarno 1998), Young Stellar Object (Mitchell et al. 1991), and interacting binary systems. Whether or not these absorption lines will be detected depends on the signal-to-noise ratio of the spectrum in combination with the column density of the observable species. This suggests that with the rise of larger telescopes and more sensitive detectors, we expect to find many more objects exhibiting circumstellar spectra.

\subsection{WHY STUDY MOLECULAR SPECTRA AS OPPOSED TO RADIO EMISSION LINES?}

Studies of radio line emission are faced with the problem that for a spatially unresolved source, the emission profile is the integrated emission of the CSE. The intensity at a given frequency, does not correspond with one location in the shell, but with a ring of material for which the projected velocity in the radial direction corresponds to that frequency. If the shell is not spherically symmetric this problem is even more complicated. Both these problems do not enter into absorption line studies: velocities are not projected and asymmetry is not important. Only inhomogeneities along the line of sight could be important.

\subsection{WHICH MOLECULAR SPECIES MIGHT BE PRESENT?}

In order to give the final answer to this question, one has to know all allowed transitions of all molecules possibly present. This is beyond the scope of our work. Instead we look at studies concerning interstellar, photospheric, and cometary molecules. Absorption lines of molecules detected in interstellar diffuse clouds are among others: $\mathrm{H}_{2}, \mathrm{HD}, \mathrm{CH}, \mathrm{CH}^{+}, \mathrm{C}_{2}, \mathrm{OH}, \mathrm{CO}, \mathrm{NH}$, 
$\mathrm{CN}, \mathrm{CS}(\mathrm{mm})$, and $\mathrm{HCl}$ (Van Dishoeck 1997). Molecules detected in the photosphere of cool stars are among others: $\mathrm{C}_{2}, \mathrm{CN}, \mathrm{CH}, \mathrm{HCN}$, and $\mathrm{C}_{2} \mathrm{H}_{2}$ (Jørgensen 1997). Molecules detected in cometary spectra are among others: $\mathrm{CO}, \mathrm{C}_{2}, \mathrm{CS}, \mathrm{NH}_{3}$ and many highly reactive species (Weaver 1997, BockeleeMorvan 1997). An alternative approach is to compute chemical models of the CSE (Cherchneff et al. 1993) and predict column densities.

The question now arises which of these molecules are likely to be present at high enough abundance to be detectable. Cometary lines are due to evaporation and successive photo-dissociation. This time scale is much shorter than the corresponding time scale in circumstellar shells and cometary molecules (especially the reactive ones) might not be abundant in circumstellar shells. Photospheric molecules exist at a much higher temperature than circumstellar molecules. The chemical equilibrium is different in the two environments and therefore different molecules might be present. Interstellar molecules experience roughly the same conditions (density, temperature, and interstellar radiation field) as circumstellar molecules. In order of probability of the presence of circumstellar molecules (with absorption spectra) we propose interstellar, followed by photospheric and cometary species. As we will demonstrated in this paper, circumstellar absorption spectra of $\mathrm{CH}^{+}, \mathrm{C}_{2}, \mathrm{CN}, \mathrm{CO}, \mathrm{C}_{3}, \mathrm{SiC}_{2}$ and emission of $\mathrm{CH}^{+}, \mathrm{C}_{2}, \mathrm{CO}$, $\mathrm{C}_{3}, \mathrm{AlO}, \mathrm{VO}$, and $\mathrm{ZrO}$ have been detected (Table 1). There remains a large amount of work to be done to check for the presence of the other molecules. In particular the detection (at high-resolution, $\lambda / \Delta \lambda \geq 30,000$ ) of circumstellar absorption of $\mathrm{H}_{2}$ and $\mathrm{CO}$ is of fundamental importance.

\subsection{WHAT CAN WE LEARN FROM MOLECULAR SPECTRA?}

Identification: A symmetric molecule like $\mathrm{C}_{2}$ does not have transitions in the radio. Electronic bands allow to study these molecule. The interstellar abundance of $\mathrm{CH}^{+}$is still not well understood, studying circumstellar $\mathrm{CH}^{+}$ spectra might help to improve our understanding of the formation process. The presence of the unidentified $21 \mu \mathrm{m}$ feature is strongly correlated with the presence of $\mathrm{C}_{2}$ and $\mathrm{CN}$ absorption, which suggests that the $21 \mu \mathrm{m}$ feature is due to a carbon bearing molecule.

Expansion velocity: Very accurate expansion velocities can be determined. The correlation of expansion velocity with the photospheric abundance pattern (mainly carbon abundance) might give information about the mass-loss mechanism. Different molecules probe different layers. The expansion velocity can be determined throughout the shell and possibly a slow change of expansion velocity can be detected as a result of the star's evolution. The temperature and chemical content of the shell can be studied as a function of radius. 
Non-LTE effects: This is important for radio emission lines since in the radio only few levels of a molecule are probed and the total number of molecules is obtained by extrapolating to higher levels given a certain LTE temperature. We found that $\mathrm{C}_{2}$ is pumped by the stellar radiation field (supra-thermal), while CN is sub-thermal (Paper I, II).

Isotope ratios: Nucleo-synthesis of AGB stars and the third dredge-up. Fig. 1 shows our first detection of ${ }^{13} \mathrm{CN}$ Violet System $(0,0)$ in HD56126 (Paper III). We found ${ }^{12} \mathrm{CN} /{ }^{13} \mathrm{CN}=23 \pm 1$ and that this ratio is affected by the isotopic exchange reaction and puts only a lower limit on the ${ }^{12} \mathrm{C} /{ }^{13} \mathrm{C}$ ratio.

Inhomogeneities (clumps): A possible change with time might be detected of the molecular bands due to inhomogeneities in the line of sight. Some of the stars pulsate. A time delay between the pulsation of the star and the change in temperature of the molecule, would yield the radius at which that molecule is present.

\section{Discussion}

Table 1 gives a summary of evolved objects for which circumstellar molecular spectra have been detected. Only those objects and molecules are listed for which there is ample evidence that they are indeed circumstellar. This could be based on the radial velocity, or on the temperature derived from the molecular band. Several groups have conducted low-resolution studies of CO (Hrivnak et al. 1994, Oudmaijer et al. 1995), $\mathrm{C}_{2}, \mathrm{CN}$, and $\mathrm{C}_{3}$ (Hrivnak 1995). These studies have not been included in this table because they do not make a distinction between photospheric and circumstellar origin. The column headed by $21 \mu \mathrm{m}$, refers to the presence of the unidentified $21 \mu \mathrm{m}$ feature, [s/Fe] refers to the photospheric abundance of s-process elements relative to $\mathrm{Fe}$, relative to the solar ratio. $\mathrm{C} / \mathrm{O}$ is the photospheric carbon over oxygen ratio.

Based on Table 1, we suggest three criteria which can be used to identify a post-AGB star: $\mathrm{A}$. the presence of $\mathrm{C}_{2}$ and $\mathrm{CN}$ absorption spectra; $\mathrm{B}$. the presence of the $21 \mu \mathrm{m}$ emission feature, and C. an enhancement of s-process elements and carbon.

Of the large range of circumstellar molecules that could exhibit molecular spectra in the ultraviolet, optical, or infrared (see Sect. 1.3), only a handful have been detected (Table 1). The detection of more molecules, but also the increase of objects exhibiting circumstellar spectra, would give valuable information on circumstellar chemistry and stellar evolution. It should be noted that in this paper we have emphasized on molecular absorption spectra and to a lesser extend molecular emission spectra. Besides molecules, there are also atoms present which will exhibit absorption lines 
TABLE 1. Circumstellar molecular lines in the optical and ultraviolet spectra of evolved stars.

\begin{tabular}{|c|c|c|c|c|c|c|c|}
\hline Post-AGB stars: & $\mathrm{C}_{2}$ & $\mathrm{CN}$ & $21 \mu \mathrm{m}$ & {$[\mathrm{s} / \mathrm{Fe}]$} & $\mathrm{C} / \mathrm{O}$ & Remarks & References \\
\hline IRAS02229+6208 & - & - & & & & & $1,1, ., .,$. \\
\hline IRAS04296+3429 & - & - & + & + & & & $2,2,4,8$, . \\
\hline IRAS05113+1347 & - & - & + & & & & $2,2,5, . .$. \\
\hline IRAS05341+0852 & - & - & + & + & + & & $2,2,5,9,9$ \\
\hline HD56126 & - & - & + & + & + & & $2,2,4,10,10$ \\
\hline IRAS07431+1115 & - & - & & & & & $1,1, ., .,$. \\
\hline IRAS08005-2356 & - & - & & & & & $2,2, ., .$, \\
\hline IRAS14429-4539 & $+?$ & & & & & & $3, ., ., .,$. \\
\hline HD187885 & nd & nd & + & + & + & & $24,24,6,11,11$ \\
\hline IRAS20000+3239 & - & - & + & & & & $2,2,5, .$. \\
\hline AFGL2688 & -+ & - & + & & & $\mathrm{SiC}_{2}$ detected & $2,2, ., .$, \\
\hline IRAS22223+4327 & - & - & + & + & + & & $2,2,5,8,8$ \\
\hline HD235858 & - & - & + & + & + & & $2,2,4,12,12$ \\
\hline IRAS22574+6609 & $-?$ & $-?$ & + & & & & $24,24,7, . .$. \\
\hline IRAS23304+6147 & - & - & + & & & & $2,2,4, ., .$. \\
\hline AGB stars: & $\mathrm{C}_{2}$ & $\mathrm{CN}$ & $21 \mu \mathrm{m}$ & {$[\mathrm{s} / \mathrm{Fe}]$} & $\mathrm{C} / \mathrm{O}$ & & \\
\hline IRC+10216 & $-?$ & - & - & + & + & & $2,2, ., 22,23$ \\
\hline Post-AGB binaries: & $\mathrm{C}_{2}$ & $\mathrm{CN}$ & $\mathrm{CH}^{+}$ & $\mathrm{CO}$ & $\mathrm{C}_{3}$ & & \\
\hline HD44179 & nd & + & + & -+ & + & & $2,19,2,20,21$ \\
\hline HD46703 & nd? & $\mathrm{nd} ?$ & nd? & & & & $24,24,24, . .$. \\
\hline HD52961 & nd & nd & nd & & & & $2,2,2, .$. \\
\hline HR4049 & nd & nd & nd & & & & $2,2,2, .,$. \\
\hline HD213985 & nd & nd & - & & & & $2,2,2, . .$. \\
\hline $\mathrm{BD}+39^{\circ} 4926$ & nd & nd & nd & & & & $2,2,2, . .$. \\
\hline R CrB stars: & $\mathrm{C}_{2}$ & $\mathrm{CN}$ & & & & & \\
\hline V854 CEN & + & + & & & & & 16,17 \\
\hline $\mathrm{R} \mathrm{CrB}$ & + & & & & & & $18,$. \\
\hline Anomalous objects: & $\mathrm{AlO}$ & $\mathrm{ScO}$ & $\mathrm{TiO}$ & $\mathrm{VO}$ & & & \\
\hline VY CMa & & + & + & & & disk? & $., 13,14,$. \\
\hline $\mathrm{U}$ Equ & + & & + & + & & disk ? & $15, ., 15,15$ \\
\hline
\end{tabular}

-: absorption; + emission or enhanced; open slots if no information available 1: Hrivnak 1998; 2: Bakker et al. 1997; 3: Hu et al. 1994; 4: Kwok et al. 1989; 5: Kwok et al. 1995; 6: Justtanont et al. 1996; 7: Hrivnak \& Kwok 1991; 8: Decin et al. 1998; 9: Reddy et al. 1997; 10: Klochkova 1995; 11: Van Winckel et al. 1996; 12: Začs et al. 1995; 13: Wallerstein 1986; 14: Phillips \& Davis 1987; 15: Barnbaum et al. 1996; 16: Rao \& Lambert 1993; 17: Withney et al. 1992; 18: Lambert et al. 1990; 19: Sarre 1996; 20: Glinski et al. 1997; 21: Glinski \& Nuth 1995; 22: Utsumi 1970; 23: Lambert et al. 1986; 24: this paper 

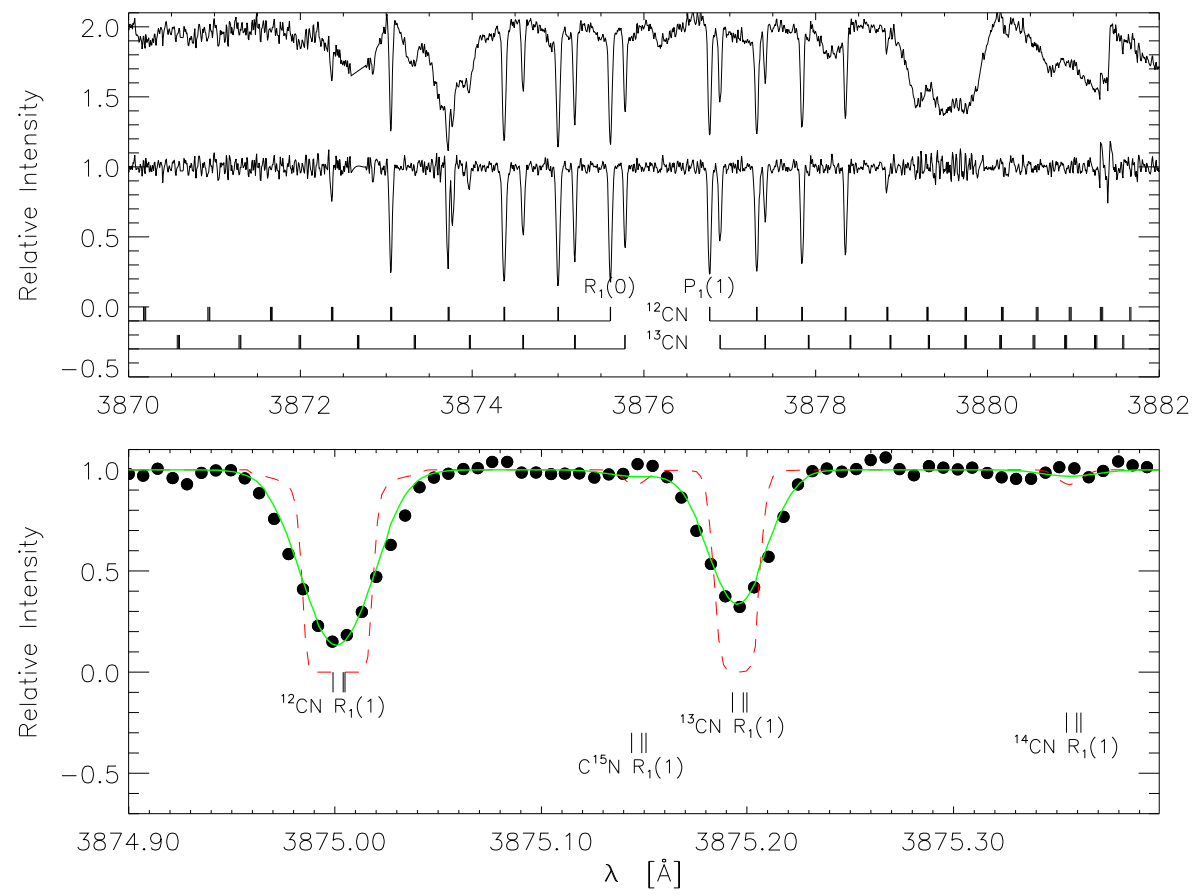

Figure 1. CN Violet System (0,0) band towards HD 56126. The upper panel shows the observed spectrum and a rectified spectrum corrected for the underlying photospheric features. The rectified spectrum contains only circumstellar lines. The lower panel shows on an expanded wavelength scale the strongest line (the $R_{1}, R_{2}$, and ${ }^{\mathrm{R}} Q_{21}$ blend for $\left.N^{\prime \prime}=1\right)$, and demonstrates that we have not detected ${ }^{14} \mathrm{CN}$ nor $\mathrm{C}^{15} \mathrm{~N}$. The dashed spectrum is a synthetic spectrum computed for $b=0.51 \mathrm{~km} \mathrm{~s}^{-1}, T_{\text {rot }}=12.6 \mathrm{~K}$ for $\mathrm{CN}, T_{\text {rot }}=8.0$ for the $\mathrm{CN}$ isotope, and the isotope or lower limit isotope ratios as determined in this work. The solid spectrum is the synthetic spectrum convolved to a spectral resolution of $R=140,000$. (Figure from Paper III).

from their ground level (Morton 1991).

In order to facilitate the search for circumstellar molecular bands, extensive line list of the relevant transitions presented in Paper I, II, and II, are (or will be) available at CDS, or can be obtained by contacting one the author.

Acknowledgments The author acknowledges the National Science Foundation (Grant No. AST-9315124) and the Robert A. Welch Foundation of Houston, Texas. This research has made use of the Simbad database, operated at CDS, Strasbourg, France, and the ADS service.

\section{References}

Bakker, E.J., Waters, L.B.F.M., Lamers, H.J.G.L.M., Trams, N.R. \& Van der Wolf, 
F.L.A. 1996, A\&A 310, 893 (Paper I)

Bakker, E.J., Van Dishoeck, E.F., Waters, L.B.F.M. \& Schoenmaker, T. 1997, A\&A, 323, 469 (Paper II)

Bakker, E.J. \& Lambert, D.L. 1998, A\&A in preparation (Paper III)

Barnbaum, C., Omont, A. \& Morris, M. 1996, A\&A 310, 259

Bockelee-Morvan, D. 1997, in "Molecules in astrophysics: probes and processes", Eds. E.F. Van Dishoeck, p219

Cherchneff, I., Glassgold, A.E. \& Mamon, G.A. 1993, ApJ 410, 188

Dalgarno, A. 1998, this volume

Decin, L., Van Winckel, H., Waelkens, C. \& Bakker, E.J. 1998, A\&A in preparation

Glinski, R.J. \& Nuth, J.A.III 1995, BAAS 186, 5701

Glinski, R.J., Nuth, J.A.III, Lauroesch, J.T., Reese, M.D. \& Sitko, M.L., 1997, BAAS 190, 1701

Hrivnak, B.J. 1995, ApJ 438, 341

Hrivnak, B.J. 1998, in preparation

Hrivnak, B.J. \& Kwok, S. 1991, ApJ 368, 564

Hrivnak, B.J., Kwok, S., Geballe, T.R. 1994, ApJ 420, 783

Hu, J.Y., Te Lintel Hekkert, P., Slijkhuis, S., Baas, F., Sahai, R. \& Wood, P.R. 1994, A\&AS 103, 301

Jørgensen, U.G. 1997, in "Molecules in astrophysics: probes and processes", Eds. E.F. Van Dishoeck, p441

Justtanont, K., Barlow, M.J., Skinner, C.J., Roche, P.F., Aitken, D.K. \& Schmith, C.H. 1996, A\&A 309, 612

Klochkova, V.G. 1995, MNRAS 272, 710

Kwok, S., Volk, K. \& Hrivnak, B.J. 1989, ApJ 345, L51

Kwok, S., Hrivnak, B.J. \& Geballe, T.R. 1995, ApJ 454, 394

Lambert, D.L., Gustafsson, B., Eriksson, K. \& Hinkle, K.H. 1986, ApJS 62, 373

Lambert, D.L., Rao, N.K. \& Giridhar, S. 1990, JApA 11, 475

Mitchell, G.F., Maillard, J.-P. \& Hasegawa, T.I., 1991, ApJ 342, 356

Morton, D.C. 1991, ApJS 77, 119

Oudmaijer, R.D., Van der Veen, W.E.C.J., Waters, L.B.F.M., Trams, N.R., Waelkens, C., Engelsman, E. 1992, A\&AS 96, 625

Phillips, J.G. \& Davis, S.P. 1987, PASP 99, 839

Rao, N.K. \& Lambert, D.L. 1993, AJ 105, 1915

Reddy, B.E., Parthasarathy, M., Gonzalez, G. \& Bakker, E.J. 1997, A\&A accepted

Utsumi, K. 1970 PASJ 22, 93

Van Dishoeck, E.F. 1997, Lecture notes, University of Texas, Austin

Van Winckel, H., Waelkens, C. \& Waters, L.B.F.M. 1996, A\&A 306, L37

Wallerstein, G. 1986, A\&A 164, 101

Weaver, H.A. 1997, in "Molecules in astrophysics: probes and processes", Eds. E.F. Van Dishoeck, p205

Whitney, B.A., Clayton, G.C., Schulte-Ladbeck, R.E. \& Maede, M.R. 1992, AJ 103, 1652

Sarre, P. 1996, Private communications

Začs, L., Klochkova, V.G. \& Panchuk, V.E. 1995, MNRAS 275, 764 